\title{
CAPACIDADE DE CARGA DE FUNDAÇÕES E VERIFICAÇÃO DE RECALQUES A PARTIR DE PARÂMETROS DO ENSAIO PANDA 2 E DE OUTROS ENSAIOS IN SITU
}

\author{
Load capacity of foundations and settlements verification from \\ parameters of panda 2 test and others in situ tests
}

\author{
Antônio Carlos Ferreira Tonhá ${ }^{1}$; Renato Resende Angelim ${ }^{2}$ \\ Recebido em 29 de julho de 2016; recebido para revisão em 05 de junho de 2017; aceito em 22 de agosto de 2017; \\ disponível on-line em 15 de setembro de 2017.
}

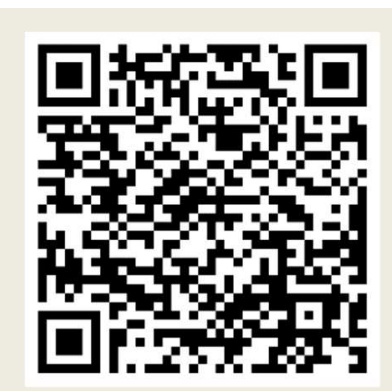

PALAVRAS CHAVE:

Ensaio PANDA;

Aterro compactado;

Capacidade de carga;

Recalque;

Fundações.

KEYWORDS:

PANDA test;

Compacted soil;

Load capacity;

Settlement;

Foundations.

* Contato com o autor:

RESUMO: O presente trabalho tem como objetivo comparar a capacidade de carga de fundações rasas, tipo sapata, usando os valores de resistência à penetração dinâmica do ensaio PANDA 2 em solo tropical compactado, em substituição aos parâmetros obtidos pelos ensaios CPT e SPT, que implementam os métodos de dimensionamento tradicionais, bem como de fundações profundas, tipo estaca. Além disso, o trabalho abrange a análise do recalque em cada uma das situações propostas. A comparação foi feita a partir do cálculo da capacidade de carga de sapata e de estaca de dimensões predefinidas por meio de métodos teóricos e semi-empíricos. Os resultados para uso do PANDA 2 foram satisfatórios, apresentando pouca variabilidade quando comparado ao uso do SPT e CPT para dimensionamento de fundações no solo estudado. Quanto aos recalques, como esperado, o recalque previsto para a estaca foi relativamente menor que o recalque para a sapata. A principal contribuição deste trabalho foi a aplicação de nova tecnologia disponível para investigação geotécnica de campo, o ensaio Panda 2, em métodos de cálculos de previsão de capacidade de carga e de recalque de fundações.

ABSTRACT: This study aims to compare the load capacity of shallow and deep foundations, spread footing and piles, using dynamic penetration resistance values of PANDA 2 test in tropical compacted soil, replacing the parameters obtained by CPT and SPT that implement the traditional methods of capacity of design load. Furthermore, this research includes also the settlements analysis in each of the situations proposed. The comparison of the load capacity of footing and pile with predefined dimensions was made through theoretical and semi-empirical methods. The PANDA 2 results use were satisfactory, with little variability when compared to SPT and CPT use for foundations design in the studied soil. With regard to settlements, as expected, the settlement for pile was relatively lower than the settlements on footing. The main contribution of this paper was the practical application of Panda 2 results use, through methods of estimate foundations load capacity and settlements.

${ }^{1}$ e-mail: antoniocftonha@gmail.com ( A. C. F. Tonhá)

Graduando em Engenharia Civil pela Escola de Engenharia Civil e Ambiental, Universidade Federal de Goiás - UFG.

2e-mail: tecnoeng@gmail.com (R. R. Angelim)

Professor, Doutor em Geotecnia, Escola de Engenharia Civil e Ambiental, Universidade Federal de Goiás - UFG. 


\section{INTRODUÇÃO}

Os tipos de fundações existentes variam desde estruturas rasas e superficiais, como por exemplo as sapatas, até fundações profundas, como as estacas, que podem atingir camadas do solo de maior capacidade de carga. Os requisitos básicos para o dimensionamento de fundações são: deformações aceitáveis sob as condições de trabalho, segurança quanto ao colapso do solo (estabilidade externa) e segurança quanto ao colapso dos elementos estruturais (estabilidade interna) (VELLOSO e LOPES, 2010).

Sob a ótica da segurança quanto ao colapso do solo, o fator determinante é a tensão induzida no solo a partir da carga incidente na fundação. A definição da fundação a ser usada, bem como o seu dimensionamento são condicionados a fatores como análise econômica, geotecnia local (tipo de solo, capacidade de carga do solo, deformabilidade), tipo de obra, características do carregamento incidente na fundação, viabilidade executiva, edificações vizinhas, o que faz com que seja indispensável a investigação geotécnica do solo para correta análise das condições gerais da obra para a definição do tipo de fundação adequado e seu dimensionamento (CINTRA, AOKI e ALBIERO, 2011).

Alguns ensaios que colaboram para os cálculos são os ensaios de laboratório para definição de parâmetros de resistência do solo (coesão e ângulo de atrito por exemplo). Os cálculos a partir destes parâmetros configuram os métodos teóricos: teorias de dimensionamento de Terzaghi para fundações rasas e de Meyerhof para fundações profundas. Ensaios de campo como o SPT (Standard Penetration Test) de penetração dinâmica e o CPT (Cone Penetration Test) de penetração estática, amparam outra metodologia de cálculo, chamada de métodos empíricos. Essa metodologia consiste na estimativa da resistência à compressão do solo a partir de correlações entre os valores destes ensaios de campo com a resistência do solo.

O ensaio mais difundido no Brasil para o dimensionamento de fundações é o SPT, apesar das incertezas que o processo executivo deste ensaio produz no que se refere à interferência do operador (anotação dos golpes e variação da energia aplicada) e do equipamento (tipo de material utilizado, uso do amostrador para escavação, entre outros) no resultado do ensaio, e na falta de padronização do processo, mesmo havendo a norma regulamentadora do SPT no Brasil, a NBR-6484 (ABNT, 2001) (SCHNAID, 2000). Entretanto, cabe salientar que ainda existem muitas obras de engenharia sendo feitas no país sem investigações geotécnicas, principalmente no que diz respeito às obras de pequeno porte.

No sentido de aumentar o leque de ensaios disponíveis para a investigação do solo, e de apresentar uma opção que pudesse baratear os serviços e facilitar o acesso a informações do solo, conferindo maior autonomia ao processo de sondagem é que se selecionou o ensaio com o equipamento PANDA 2, desenvolvido a partir do aperfeiçoamento do PANDA, criado em 2000, na França (SOL SOLUTION, 2009).

O PANDA 2 apresenta vantagens como, por exemplo leveza, portabilidade e facilidade de execução, além de fornecer as características do perfil do solo quanto à resistência à penetração em avanços inferiores a $20 \mathrm{~mm}$ por golpe. Entretanto, para que ele possa ser utilizado para fins de sondagem para projeto de fundações, é importante que esse ensaio, tenha seus resultados correlacionados com os resultados de ensaios já consagrados, como o SPT e o CPT, dando assim maior segurança ao projetista no uso de seus resultados.

Pesquisas realizadas na Universidade Federal de Goiás como as de Alves e Silva (2009), Ferreira, Quirino e Soares (2013), Azevedo e Rodrigues (2014), Cruz Jr. Et al. (2014) e Angelim et al. (2016a) comparando o SPT e o ensaio PANDA 2 em solos tropicais mostraram viabilidade do uso do PANDA 2 em sondagens para fundações. Dessa forma, este estudo justifica-se pela aplicação tecnológica dos resultados desse novo e não convencional equipamento de investigação geotécnica em métodos consagrados de determinação da capacidade de carga e de recalques de fundações em sapata e em estaca. 


\section{OBJETIVOS}

O objetivo deste trabalho é determinar a capacidade de carga de uma sapata e de uma estaca com dimensões predefinidas, utilizando métodos teóricos e empíricos/semi-empíricos por meio de resultados de ensaios SPT e PANDA 2, bem como a previsão de recalques para cada uma das situações estudadas. Além disso, realizar uma análise comparativa entre os resultados obtidos pelos diferentes métodos.

\section{REVISÃO BIBLIOGRÁFICA}

\subsection{RELAÇÃO ENTRE SPT E CPT}

Velloso e Lopes (2010), relatam que entre os principais processos de investigação do subsolo para fins de projeto de fundações de estrutura encontram-se as sondagens a percussão (SPT) e o ensaio de cone (CPT).
Velloso e Lopes (2010) correlacionaram, em seu estudo, a resistência à penetração do cone (qc) do ensaio CPT com a resistência à penetração (NSPT) do ensaio SPT através da Equação 1. Os valores de " $k$ " estão dispostos na Tabela 1 , onde estão apresentados também os valores para o coeficiente $\alpha$, denominado razão de atrito. Este parâmetro relaciona o atrito lateral do cone ( $\mathrm{fs}$ ) com a tensão de ponta (qc) determinados no ensaio CPT e variam de acordo com o tipo de solo atravessado pelo cone. Cabe salientar que o valor de NSPT da Equação 1, considera uma eficiência de energia do ensaio SPT dentro da média brasileira, em torno de $72 \%$.

$$
\mathrm{q}_{\mathrm{c}}=\mathrm{k} \mathrm{N}_{\mathrm{SPT}}
$$

Em que:
$\mathbf{q}_{\mathrm{c}}=$ resistência à penetração do cone $(\mathrm{MPa})$;
$\mathbf{k}=$ coeficiente de correlação entre $\mathrm{N}_{\mathrm{SPT}}$ e $\mathrm{q}_{\mathrm{c}}$;
$\mathbf{N}_{\text {SPT }}=$ número de golpes do ensaio SPT .

TABELA 1: Valores de coeficiente $k$ para $q_{c}$ em MPa, e valores da razão de atrito $\alpha$ para dimensionamento de estacas via método Aoki-Velloso.

\begin{tabular}{ccc} 
Solo & $\mathbf{k}$ & $\boldsymbol{\alpha ~ ( \% ) ~}$ \\
\hline Areia & 1,00 & 1,4 \\
\hline Areia siltosa & 0,80 & 2,0 \\
\hline Areia siltoargilosa & 0,70 & 2,4 \\
\hline Areia argilosa & 0,60 & 3,0 \\
\hline Areia argilossiltosa & 0,50 & 2,8 \\
\hline Silte & 0,40 & 3,0 \\
\hline Silte arenoso & 0,55 & 2,2 \\
\hline Silte arenoargiloso & 0,45 & 2,8 \\
\hline Silte argiloso & 0,23 & 3,4 \\
\hline Silte argiloarenoso & 0,25 & 3,0 \\
\hline Argila & 0,20 & 6,0 \\
\hline Argila arenosa & 0,35 & 2,4 \\
\hline Argila arenossiltosa & 0,30 & 2,8 \\
\hline Argila siltosa & 0,22 & 4,0 \\
\hline Argila siltoarenosa & 0,33 & 3,0 \\
\hline & & FONTE: Aoki e Velloso (1975).
\end{tabular}




\subsection{ENSAIO PANDA 2}

O PANDA (Pénétromètre Autonome Numérique Dynamique Assisté par Ordinateur), é um equipamento de sondagem o subsolo a partir da penetração dinâmica de ponteira cônica, desenvolvido pela empresa francesa Sol Solution, com a finalidade de ser um equipamento leve e portátil para investigações geotécnicas de campo, além disso, pode ser usado também como alternativa no controle de compactação de solos (SOL SOLUTION, 2009).

O PANDA 2, sua versão mais recente, está apresentada na Figura 1 e trata-se de um penetrômetro de cone que funciona com energia variável, que é calculada instantaneamente no momento do golpe por uma célula de carga no capacete. Os deslocamentos de cravação são precisamente registrados e utilizados para o cálculo do seu parâmetro de resistência dinâmica à penetração do cone $\left(q_{d}\right)$, minimizando assim a interferência do operador e do próprio equipamento nos resultados do ensaio.

O mecanismo de medição da resistência de ponta " $q_{d}$ " consiste em usar um martelo de massa conhecida para golpear um capacete de metal que fica sobre um conjunto de hastes metálicas com uma ponteira cônica em contato com o solo, que ao receber o impacto, penetra no mesmo. A velocidade de queda do martelo é medida logo em seguida ao impacto deste com o capacete. Uma unidade central de aquisição, apoiada na superfície do terreno, ligada ao capacete por uma correia denteada (trena) registra a profundidade de penetração das hastes no solo. Os dados de energia e de profundidade de cravação são armazenados em um terminal de comunicação e processamento, que calcula o valor de resistência dinâmica à penetração. $O$ cálculo é função da área de ponta (2, 4 ou $10 \mathrm{~cm}^{2}$ ) e do número de hastes, de massas conhecidas, utilizadas no ensaio.

A Equação 2 mostra a fórmula dos Holandeses para se calcular o valor da resistência de ponta (SOL SOLUTION, 2009):

$$
\mathrm{q}_{\mathrm{d}}=\frac{1}{\mathrm{~A}} \frac{1 / 2 M V}{(1+P / M)} \frac{1}{\mathrm{e}}
$$

\section{Em que:}

$\mathbf{q}_{\mathbf{d}}=$ resistência à penetração dinâmica ou resistência de ponta $(\mathrm{Pa})$;

$A=$ área da ponta de cone $\left(\mathrm{m}^{2}\right)$;

$\mathbf{M}=$ massa do martelo $(\mathrm{kg})$;

$\mathbf{V}=$ velocidade do impacto $(\mathrm{m} / \mathrm{s})$

$\mathbf{P}=$ massa das hastes e ponta $(\mathrm{kg})$;

$\mathbf{e}=$ penetração da ponta $(\mathrm{m})$.
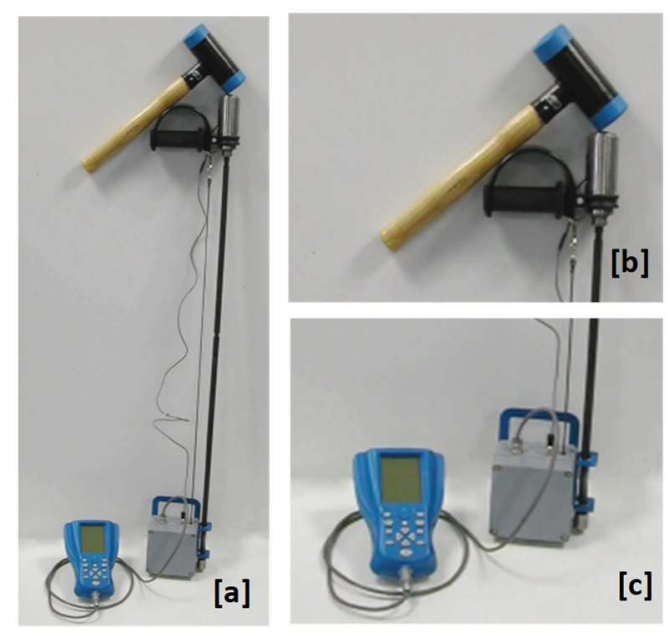

FIGURA 1: Equipamento PANDA 2: [a] Equipamento completo; [b] Detalhe da cabeça de bater e do martelo; [c] Detalhe do terminal de comunicação (à esquerda) e da central de aquisição (à direita).

FONTE: Sol Solution (2009). 
Ferreira, Quirino e Soares (2013) e Angelim et al. (2016a) trataram os dados dos ensaios PANDA 2 e SPT de Angelim (2011) que foram realizados em aterro compactado de solo argiloso em ombreira de barragem de terra, com teor de umidade in situ entre $15 \%$ e $20 \%$, e verificaram que para profundidades de até 5,0 metros os resultados dos ensaios PANDA $2 \mathrm{com}$ ponteira de $4 \mathrm{~cm}^{2}$, e os dos ensaios SPT puderam ser satisfatoriamente correlacionados (com coeficiente de variação igual 7,62\%) por meio da Equação 3:

$$
\mathrm{q}_{\mathrm{d}}=0,26 \mathrm{~N}_{\mathrm{SPT}}
$$

\section{Em que:}

$\mathbf{q}_{d}=$ resistência de ponta do PANDA 2;

$\mathbf{N}_{\text {SPT }}=$ número de golpes do ensaio SPT.

\subsection{DETERMINAÇÃO DA CAPACIDADE DE CARGA DAS FUNDAÇÕES}

Velloso e Lopes (2010) definem capacidade de carga de uma fundação como sendo a carga com a qual o solo sob essa fundação se rompe. A NBR 6122, projeto e execução de fundações, (ABNT, 2010) normaliza o projeto e a execução de fundações no Brasil, e se divide basicamente em dois grandes grupos: as fundações rasas e as fundações profundas.

\subsubsection{Fundações rasas}

Segundo Teixeira e Godoy (1998), o dimensionamento de fundações diretas se dá por meio da estimativa prévia da tensão admissível do solo, e consequente dimensionamento da área necessária para que seja distribuída a carga gerada pelo pilar na fundação, conforme a Equação 4:

$$
\mathrm{A}=\frac{\mathrm{P}}{\sigma_{\mathrm{a}}}=\mathrm{BL}
$$

\section{Em que:}

$$
\text { A = Área necessária }\left(\mathrm{m}^{2}\right) \text {; }
$$

\footnotetext{
${ }^{1}$ TERZAGHI, K. Theoretical soil mechanics. New York:
}

$\mathbf{P}=$ Solicitação de projeto $(\mathrm{kN})$;

$\boldsymbol{\sigma}_{\mathrm{a}}=$ Tensão admissível do solo $\left(\mathrm{kN} / \mathrm{m}^{2}\right)$;

$\mathbf{B}$ e $\mathbf{L}=$ Largura e comprimento da sapata retangular $(\mathrm{m})$.

Deste modo, se torna imprescindível que se determine a tensão admissível do solo, que pode ser calculada por meio de diferentes metodologias que variam de acordo com o parâmetro geotécnico característico do local, obtido pela investigação geotécnica realizada. Os métodos podem ser baseados em ensaios de laboratório que fornecem o ângulo de atrito, peso específico e coesão do solo em estudo ou baseados em ensaios in situ como SPT e CPT. Ressalta-se ainda, que segundo a NBR 6122 (ABNT, 2010), a tensão admissível da fundação (qad) se caracteriza pela tensão última da fundação (ruptura) que deve ser reduzida por um fator de segurança (F.S.) de valor 3 para as fundações superficiais.

O Método de Terzaghi $(1943)^{1}$, propõe a Equação 5, deduzida para sapata quadrada, desprezando a resistência ao cisalhamento do solo acima do nível da base, substituindo-o por uma sobrecarga (YD) e considerando solo homogêneo.

$$
\mathrm{q}_{\mathrm{Ult}}=1,3 \mathrm{c} \mathrm{N} \mathrm{c}_{\mathrm{c}}+\gamma \mathrm{D} \mathrm{N}_{\mathrm{q}}+0,8 \gamma \frac{\mathrm{B}}{2} \mathrm{~N}_{\gamma} \text { Eq. [5] }
$$

\section{Em que:}

qult = Tensão última $(\mathrm{kPa})$;

$\mathbf{B}=$ base da fundação $(\mathrm{m})$;

$\mathbf{c}=$ coesão do solo $(\mathrm{kPa})$;

$\mathbf{Y}=$ peso específico do solo $(\mathrm{kPa})$;

$\mathbf{D}$ = camada de solo acima do nível da base da sapata $(\mathrm{m})$;

$\mathbf{N c}, \mathbf{N q}$ e $\mathbf{N Y}=$ fatores de capacidade de carga fornecidos a partir de gráfico (clássico da Teoria de Terzaghi) em função do ângulo de atrito do solo.

Dentro dos métodos empíricos, Teixeira e Godoy (1998) e Cintra, Aoki e Albiero (2011) indicam o cálculo da tensão admissível para sapatas por meio da Equação 6, utilizando o valor de NSPT, e

John Wiley \& Sons, 1943. 
pelas Equações 7 e 8, utilizando valores de qc $\geq 1,5$ MPa do ensaio CPT, com tensão admissível expressa em MPa.

O valor de NSPT e qc nas equações tratam se do valor médio ao longo do bulbo de tensões, considerado 1,5 vezes o valor da menor largura da sapata (B).

- Válida para qualquer solo natural no intervalo de $4 \leq N_{S P T} \leq 20$.

$\sigma_{\mathrm{a}}=\frac{\mathrm{N}_{\mathrm{SPT}}}{50} \leq 0,4 M P a$

- Areias:

$\sigma_{\mathrm{a}}=\frac{\mathrm{q}_{\mathrm{c}}}{15} \leq 0,4 M P a$

- Argilas:

$$
\sigma_{\mathrm{a}}=\frac{\mathrm{q}_{\mathrm{c}}}{10} \leq 0,4 M P a
$$

\subsubsection{Fundações profundas}

Cintra e Aoki (2010) explicam que a capacidade de carga de uma estaca é definida como a soma das tensões resistentes por adesão ou atrito lateral em toda a área do fuste da estaca com a tensão resistente normal à base pela área da base ou ponta da estaca, como representados nas Equações 9 a 11.

$$
\begin{array}{ll}
\mathrm{R}=\mathrm{R}_{\mathrm{L}}+\mathrm{R}_{\mathrm{P}} & \text { Eq. [9] } \\
\mathrm{R}_{\mathrm{L}}=\mathrm{r}_{\mathrm{L}} \mathrm{A}_{\mathrm{L}} & \text { Eq. [10] } \\
\mathrm{R}_{\mathrm{P}}=\mathrm{r}_{\mathrm{P}} \mathrm{A}_{\mathrm{P}} & \text { Eq. [11] }
\end{array}
$$

Em que:

$\mathbf{R}=$ capacidade de carga da estaca $(\mathrm{kN})$;

$\mathbf{R}_{\mathbf{P}}=$ resistência de ponta $(\mathrm{kN})$;

$\mathbf{R}_{\mathbf{L}}=$ resistência lateral $(\mathrm{kN})$;

$r_{p}=$ tensão resistente na base $\left(\mathrm{kPa}\right.$ ou $\left.\mathrm{kN} / \mathrm{m}^{2}\right)$;

$\mathbf{r}_{\mathrm{L}}=$ tensão resistente por adesão ou atrito lateral $\left(\mathrm{kPa}\right.$ ou $\left.\mathrm{kN} / \mathrm{m}^{2}\right)$;

$A_{P}=$ área da ponta da estaca $\left(\mathrm{m}^{2}\right)$;

\footnotetext{
${ }^{2}$ AOKI, N.; VELLOSO, D. A. An Approximate method to estimate the
} bearing capacity of piles. In: PANAMERICAN CONFERENCE ON SOIL
$A_{L}=$ área lateral da estaca $\left(m^{2}\right)$

A NBR-6122 (ABNT, 2010) normaliza o fator de segurança (F.S.) com valor de 2 para determinação da carga admissível $\left(q_{a d}\right)$ de fundações profundas.

Dentre os métodos semi-empíricos para dimensionamento de estacas, se destacam alguns métodos brasileiros, como por exemplo o método de Décourt-Quaresma (1978) e o de Aoki-Velloso $(1975)^{2}$

O método de Aoki-Velloso correlaciona a tensão de ruptura de ponta ( $\left.q_{p}\right)$ e a tensão de ruptura lateral $\left(q_{L}\right)$ a partir de " $q_{c}$ " do ensaio CPT conforme mostrado nas Equações 12 e 13 (expressas em MPa), utilizando fatores de correção $\mathrm{F} 1$ e $\mathrm{F} 2$, respectivamente, que levam em conta o efeito escala, ou seja, a diferença de comportamento entre a estaca (protótipo) e o cone do CPT (modelo) e também a influência do método executivo de cada tipo de estaca. Para o caso de estaca hélice-contínua deste estudo, serão utilizados $F_{1}=2$ e $F_{2}=2 F 1$, valores esses atualizados por Cintra e Aoki (2010). Além disso, a Equação 13 faz uso da razão de atrito $(\alpha)$, já apresentada na Tabela 1.

$$
q_{\mathrm{P}}=\frac{\mathrm{q}_{\mathrm{c}}}{\mathrm{F}_{1}}
$$

$$
q_{L}=\alpha \frac{\mathrm{q}_{\mathrm{c}}}{\mathrm{F}_{2}}
$$

Já no método de Décourt-Quaresma, utiliza se para o cálculo da resistência de ponta e da resistência lateral as Equações 14 e 15, respectivamente:

$$
q_{\mathrm{P}}=\mathrm{C} \mathrm{N}^{\prime}
$$

\section{Em que:}

$\mathbf{q}_{\mathrm{P}}=$ resistência de ponta $(\mathrm{kPa})$;

$\mathbf{N}^{\prime}$ = a média dos valores de $\mathrm{N}_{\mathrm{SPT}}$ imediatamente

MECHANICS AND FOUNDATIONS ENGENEERING, 5., 1975, Buenos Aires. 
anterior a ponta da estaca, na ponta da estaca e o imediatamente posterior;

$\mathbf{C}=$ tensão de proporcionalidade (kPa), apresentada na Tabela 2 .

$$
q_{\mathrm{S}}=10\left(\frac{N_{\text {barra }}}{3}+1\right)
$$

Em que:

$\mathbf{q}_{\mathrm{s}}=$ resistência lateral $(\mathrm{kPa})$;

$\mathbf{N}_{\text {barra }}=$ é a média dos valores de $\mathrm{N}_{\mathrm{SPT}}$ ao longo do fuste da estaca, desconsiderando os valores usados no cálculo da resistência de ponta. Valores menores que 3 são assumidos como 3 e valores acima de 50 devem ser assumidos como 50 (VELLOSO e LOPES, 2010).

TABELA 2: Valores da Tensão de proporcionalidade " $C$ "

\begin{tabular}{cc} 
Tipo de solo & C (kPa) \\
\hline Argilas & 120 \\
\hline Siltes argilosos (alteração de rocha) & 200 \\
\hline Siltes arenosos (alteração de rocha) & 250 \\
\hline Areias & 400
\end{tabular}

FONTE: Décourt e Quaresma (1978).

O método teórico, conhecido como método de Meyerhof (1951) ${ }^{3}$ apresenta uma teoria clássica para o cálculo da capacidade de carga de estaca em solo homogêneo. Este dimensionamento consiste nos cálculos das resistências de ponta e lateral a partir das Equações 16 e 17:

$$
\begin{array}{ll}
q_{\mathrm{P}}=1,3 \mathrm{c} \mathrm{N}_{\mathrm{c}}+\frac{\mathrm{K}_{\mathrm{S}}}{2} \mathrm{q} \mathrm{N}_{\mathrm{q}} & \text { Eq. [16] } \\
q_{L}=\mathrm{a}+\mathrm{K}_{0} \mathrm{q} \tan \delta & \text { Eq. [17] }
\end{array}
$$

Em que:

$$
\begin{aligned}
& \mathbf{q}_{\mathbf{p}}=\text { resistência de ponta }(\mathrm{kPa}) ; \\
& \mathbf{q}_{\mathbf{L}}=\text { resistência lateral }(\mathrm{kPa}) ; \\
& \mathbf{c}=\text { coesão do solo }(\mathrm{kPa}) ; \\
& \mathbf{a}=\text { adesão do solo à estaca, dada por " } 2 / 3 \mathrm{c} \text { ” para } \\
& \text { estacas sem deslocamento }(\mathrm{kPa}) ;
\end{aligned}
$$

\footnotetext{
${ }^{3}$ MEYERHOF, G. G. The ultimate bearing capacity of foundations,
}

$q=\Upsilon_{\text {nat }} z\left(k N / m^{3}\right)$;

$\mathbf{K}_{\mathbf{s}}=\mathbf{K}_{\mathbf{0}}=$ coeficiente de empuxo no repouso do solo, quando não disponível, sugere-se o uso de (1sen $\phi)$ para areias e siltes e (0,95-sen $\phi)$ para argilas normalmente adensadas;

$\tan \delta=\tan (2 \phi / 3)$;

$\mathbf{N}_{\mathbf{c}}$ e $\mathbf{N}_{\mathbf{q}}=$ fatores de carga obtidos a partir de gráficos (clássicos da Teoria de Meyerhof) em função do ângulo de atrito.

\subsection{DETERMINAÇÃO DE RECALQUES}

Ao ser carregado e transferir os carregamentos para o solo, o elemento de fundação sofre recalque. Esse fenômeno se processa basicamente em duas partes: um recalque imediato e outro com o passar do tempo (Velloso e Lopes, 2010).

Os recalques que ocorrem com o passar do tempo são os recalques devidos aos fenômenos de adensamento (saída da água dos poros do solo e a redução do índice de vazios) e fenômenos viscosos (fluência), conhecido como recalque secundário (Velloso e Lopes, 2010).

De acordo com Cintra (2011) os recalques imediatos podem ser calculados por meio da Teoria da Elasticidade. Apesar de os solos não serem materiais elásticos e consequentemente, esses recalques não serem completamente reversíveis com o descarregamento, é razoável admissão do comportamento linear da curva de carga $\mathrm{x}$ recalque até níveis de tensão suficientemente distantes da ruptura. Neste trabalho serão tratados apenas os recalques imediatos.

Para fundações de compressão, Velloso e Lopes (2010) apresenta o método por camadas para o cálculo de recalques. Esse método consiste em dividir o perfil do terreno em subcamadas em função do tipo do material (camadas) ou da variação do estado de tensões (espraiamento). Depois de dividir o subsolo em subcamadas, é calculado o acréscimo de tensão $\Delta \sigma$ no ponto médio de cada subcamada. A partir do acréscimo de tensão de cada subcamada, do módulo de elasticidade do solo e da espessura da subcamada pode ser calculado o 
recalque referente à subcamada, conforme a Equação 18. O recalque total será a soma dos recalques de todas as subcamadas, conforme a Equação 19.

$$
\begin{aligned}
& \Delta \rho=\frac{\Delta \sigma}{E_{S}} \Delta h \\
& \rho=\sum \Delta \rho
\end{aligned}
$$

Em que:

$\boldsymbol{\rho}=$ Recalque total $(\mathrm{m})$;

$\Delta \boldsymbol{\rho}=$ Recalque no ponto médio da subcamada $(\mathrm{m})$;

$\Delta \boldsymbol{\sigma}=$ Acréscimo de tensões no ponto médio da subcamada ( $\mathrm{kPa})$;

$\boldsymbol{\Delta} \mathbf{h}=$ Espessura da subcamada $(\mathrm{m})$;

$E_{s}=$ Módulo de elasticidade do solo $(\mathrm{kPa})$.

O cálculo do acréscimo de tensões no ponto médio da camada pode ser feito a partir do método desenvolvido por Fadum, que consiste na determinação de um fator " $\mid$ ", dependente de fatores $\mathrm{m}$ e $\mathrm{n}$ para o centro da sapata, conforme Equação 20.

$$
\Delta \sigma=4 I q_{0} \quad \text { Eq. [20] }
$$

Em que:

$\Delta \boldsymbol{\sigma}=$ Acréscimo de tensões no ponto médio da subcamada;

I = Fator de Influência;

$\mathbf{q}_{0}=$ carga sobre o ponto de análise $\left(\Upsilon_{\text {nat }} \times \Delta h\right)$;

$\mathbf{m}=\mathbf{n}=0,5 B$ (para as sapatas quadradas de lado $B$ )

dividido pela profundidade da base ao ponto médio da subcamada.

Já a metodologia de cálculo de recalque para um único elemento de fundação por estaca, desenvolvida por Poulos e Davis (1974), se baseia na Equação 21:

$$
\rho=\frac{P I_{0} R_{K} R_{H} R_{v}}{E_{s} D}
$$

Em que:

$\boldsymbol{\rho}=$ Recalque imediato da estaca;

$\boldsymbol{P}=$ Carga atuante na estaca;

$I_{0}=$ Fator de influência;
$\boldsymbol{R}_{K}=$ Fator de correção para compressibilidade da estaca;

$\boldsymbol{R}_{\boldsymbol{H}}=$ Fator de correção para camada de profundidade finita do solo até a base rígida;

$\boldsymbol{R}_{\boldsymbol{v}}=$ Fator de correção para coeficiente de Poisson do solo;

$E_{s}=$ Módulo de elasticidade do solo;

$\boldsymbol{D}=$ Diâmetro da estaca.

\section{METODOLOGIA}

\subsection{CARACTERIZAÇÃO DA ÁREA DA PESQUISA}

O presente trabalho foi realizado com base nos estudos prévios de Angelim (2011) na barragem do Ribeirão João Leite, situada em Goiânia GO. A obra possui 389 m de extensão em concreto compactado com rolo e $62 \mathrm{~m}$ de aterro referente às duas ombreiras. A altura máxima é de $52 \mathrm{~m}$, com crista posicionada na cota $752,50 \mathrm{~m}$. A área inundada é de aproximadamente $10,4 \mathrm{~km}^{2}$, na cota 749,00 m referente ao N.A. máximo normal. A principal finalidade da obra foi o abastecimento público de água para a região metropolitana de Goiânia. A referida pesquisa estudou o aterro compactado da ombreira esquerda, à jusante da barragem. Este local foi escolhido para a coleta de amostras e para realização de ensaios in situ por se tratar de um platô de fácil acesso pós obra, permitindo futuros ensaios no campo experimental.

Para este trabalho foram utilizados os resultados dos ensaios no aterro de solo tropical compactado da barragem, sabendo que para a maioria dos casos práticos de fundação o estudo é realizado em solo natural. Entretanto, acredita-se que a escolha do aterro de solo compactado trouxe como vantagem o fato de ter sido construído com rigoroso controle de qualidade, que tornou o perfil relativamente homogêneo, em se tratando de solos, comparado ao solo natural, aliando-se ao fato de que este solo já foi bastante estudado por Angelim (2011), Angelim et al. (2016a) e Angelim et al. (2016b).

\subsection{AMOSTRAGTEM E ENSAIOS DE LABORATÓRIO}

Na Tabela 3 estão apresentados alguns parâmetros do solo que foram utilizados para os 
cálculos. Em amostras deformadas e indeformadas coletadas a cada metro de profundidade nos $8 \mathrm{~m}$ do perfil, durante a construção do aterro, Angelim (2011) realizou ensaios de caracterização e ensaios triaxiais $C D_{\text {nat }}$ e $C D_{\text {sat }}$, além de ensaio triaxial $K_{0}$. Os resultados apresentados são os médios do perfil.

\subsection{PROCEDIMENTOS DOS ENSAIOS IN SITU}

Segundo Angelim et al. (2016a) os ensaios SPT 1 e 2 foram feitos de forma contínua, ou seja, com avanço do furo em $10 \mathrm{~cm}$ e uma nova determinação de NSPT, com o objetivo de se obter mais resultados ao longo do perfil, enquanto que o SPT 3 foi realizado da forma convencional, ou seja, uma determinação de NSPT por metro, conforme a NBR-6484, sondagens de simples reconhecimento com SPT (ABNT, 2001).
Ensaios PANDA 2 foram realizados ao lado de cada um dos furos de SPT. Ao lado do furo SPT 1 foram feitas três sondagens PANDA 2, denominadas P1A, P1B e P1C, ao lado de SPT 2 outras três denominadas $\mathrm{P} 2 \mathrm{~A}, \mathrm{P} 2 \mathrm{~B}$ e $\mathrm{P} 2 \mathrm{C}$ e ao lado do furo SPT 3 apenas duas, denominadas P3A e P3C.

Angelim (2011) e Angelim et al. (2016b) apresentaram os resultados de 3 furos de sondagem com pressiômetro Ménard, sendo que em cada furo foram realizados 8 ensaios pressiométricos, a cada metro de profundidade do perfil. No furo 1 obtevese uma média para o módulo Menard (EM), o valor de $14 \mathrm{MPa}$, com coeficiente de variação $(\mathrm{CV})=$ $26,6 \%$, para o furo $2, \mathrm{EM}=18 \mathrm{MPa}$ e $\mathrm{CV}=10,5 \%$ e para o furo 3, EM = $14 \mathrm{MPa}$ e $\mathrm{CV}=18 \%$. Os autores sugeriram para o maciço o valor médio de $\mathrm{EM}=15$ $\mathrm{MPa}$ (CV geral $=21,2 \%$ ).

TABELA 3: Valores dos parâmetros do solo em estudo a partir de ensaios de laboratório.

\begin{tabular}{|c|c|}
\hline Parâmetro & Valor \\
\hline Peso específico natural médio $-\Upsilon_{\text {nat }}\left(\mathrm{kN} / \mathrm{m}^{3}\right)$ & 19 \\
\hline Peso específico seco médio $-\Upsilon_{d}\left(\mathrm{kN} / \mathrm{m}^{3}\right)$ & 16 \\
\hline Intervalo de umidade do perfil - $\mathbf{W}$ in situ (\%) & $19,1-22,2$ \\
\hline Intervalo de saturação do perfil - S in situ (\%) & $70,5-93,9$ \\
\hline Coeficiente de empuxo em repouso - Ko & 0,48 \\
\hline Coeficiente de Poisson ( $v$ ) & 0,33 \\
\hline Coesão via $C D_{\text {nat }}-c^{\prime}(\mathrm{kPa})$ & 107 (coeficiente de variação = 30,3\%) \\
\hline Coesão via $\mathrm{CD}_{\text {sat }}-\mathrm{c}^{\prime}(\mathrm{kPa})$ & 50 (coeficiente de variação = 30,8\%) \\
\hline Ângulo de atrito via $C D_{\text {nat }}\left({ }^{\circ}\right)$ & 30 \\
\hline Ângulo de atrito via $C D_{\text {sat }}\left({ }^{\circ}\right)$ & 32,5 \\
\hline Classificação granulométrica (com defloculante) & Argila arenosa ( 2 frações preponderantes) \\
\hline Classificação granulométrica (sem defloculante) & Areia siltosa (2 frações preponderantes) \\
\hline Classificação unificada - SUCS (com defloculante) & CL (Argila de baixa plasticidade) \\
\hline Classificação unificada - SUCS (sem defloculante) & SM (Areia siltosa) \\
\hline Classificação rodoviária - TRB & A 7-6 (IG=11) (Solo argiloso) \\
\hline
\end{tabular}




\subsection{METODOLOGIA PARA CONFRONTAÇÃO ENTRE SPT E PANDA 2}

Os ensaios PANDA 2 geraram uma grande quantidade de resultados de resistência à penetração $\left(q_{d}\right)$ ao longo da profundidade com a aquisição automática e milimétrica. Desta forma, foi necessário fazer um tratamento das amostras de dados, de forma a selecionar apenas os dados de resistência $\left(q_{d}\right)$ dos intervalos de $30 \mathrm{~cm}$, referentes a posição dos dois últimos $15 \mathrm{~cm}$ da determinação de $N_{S P T}$. $O$ valor médio de resistência à penetração deste trecho de $30 \mathrm{~cm}$ foi denominado de " $\mathrm{q}_{\mathrm{d} 30}$ ", para que a comparação entre os dois métodos fosse coerente. Por convenção, os valores de " $\mathrm{q}_{\mathrm{d} 30}$ ", foram posicionados no perfil no ponto médio destes $30 \mathrm{~cm}$ ao longo da profundidade, conforme está apresentado nas Tabelas 4 a 6.

Devido a semelhança entre os resultados dos ensaios Panda 2 realizados em torno do mesmo furo SPT (Angelim et al. 2016a) e para simplificar a comparação foi feita a média dos resultados dos ensaios Panda 2 (Exemplo: $P 1$ é a média de $P 1_{A}, P 1_{B}$ e P1c), como apresentado nas Tabelas de 4 a 6.

A Equação 1 foi utilizada para transformar o $N_{S P T}$ em resistência a penetração de cone $\left(q_{c}\right)$ do ensaio CPT. Para uso dessa equação, utilizou-se o valor de $\mathrm{k}$ igual a 0,20 , retirado da Tabela 1 , de acordo com o tipo de solo (no caso: argila) determinado pela classificação granulométrica apresentada na Tabela 3.

\subsection{MÉTODOS DE CÁLCULO}

De posse dos resultados dos ensaios, foi estabelecido que seria determinada a capacidade de carga de uma sapata e uma estaca de dimensões pré-definidas por meio de variadas metodologias de cálculo, apresentadas a seguir:

- $\quad$ Sapata, pelo método teórico de Terzaghi e empíricos baseados no SPT e CPT, substituindo " $\mathrm{q}_{c}$ " do CPT por " $\mathrm{q}_{d}$ " do ensaio Panda 2;

- $\quad$ Estaca, pelos métodos teórico de Meyerhof e semi-empíricos de Décourt-Quaresma e de Aoki-Velloso, sendo que neste último substituiu-se " $\mathrm{q}_{\mathrm{c}}$ " do CPT por " $\mathrm{q}_{\mathrm{d}}$ " do ensaio Panda 2, além da conversão de $\mathrm{N}_{\mathrm{SPT}}$ em $\mathrm{q}_{\mathrm{c}}$, como também propõe o método.
Foi definida então a geometria de uma sapata quadrada com 2 metros de lado e base assente a $1 \mathrm{~m}$ de profundidade e de uma estaca tipo hélice contínua até $6 \mathrm{~m}$ de profundidade, desprezando para fins de capacidade de carga o primeiro metro próximo a superfície, e $c 0 m 50 \mathrm{~cm}$ de diâmetro.

Usando as fórmulas apresentadas no item 3.3 calculou-se as cargas admissíveis de projeto que a sapata e a estaca suportariam de acordo com cada um dos métodos propostos anteriormente. Essas cargas estão apresentadas na Tabela 7 e 8.

Usando o valor da resistência à penetração dinâmica do cone " $q_{d}$ " (do Panda 2) como a resistência a penetração estática " $\mathrm{q}_{\mathrm{c}}$ " nos métodos que utilizam CPT, pôde-se aplicar os resultados do PANDA 2 nas equações dos métodos que utilizam os resultados de CPT, complementando as Tabelas 7 e 8.

Após realizar os cálculos de capacidade de carga de cada elemento de fundação a partir dos métodos propostos, foi determinada a previsão de recalque teórico para cada situação, conforme item 3.4. Para a sapata foi utilizado a metodologia de camadas (Eq. 18 e Eq. 19), calculando o acréscimo de tensões a partir do Fadum (Eq. 20). Os recalques das estacas foram calculados de acordo com o método de Poulos e Davis apresentado pela Eq. 21.

Para cada carga admissível foi determinada a previsão do recalque correspondente. Foi utilizado o valor médio de 15 MPa para o módulo de elasticidade do solo obtido in situ com o Pressiômetro Ménard.

$\mathrm{Na}$ verificação dos recalques, foi considerado para o elemento de fundação em estaca, concreto de fck $=20 \mathrm{MPa}$ e módulo de elasticidade $\left(E_{p}\right.$ ) estimado de $28,85 \mathrm{GPa}$, bem como $45 \mathrm{~m}$ para a profundidade da camada de solo até a rocha sã $(H)$, conforme indicação do projeto do barramento.

\section{ANÁLISE E DISCUSSÃO DOS RESULTADOS}

A Tabela 4 apresenta os resultados médios dos ensaios Panda 2 realizados em torno do furo do SPT3. Este ensaio foi realizado de forma 
convencional (a cada metro), conforme NBR-6484 (ABNT, 2001).

As Tabelas 5 e 6 apresentam os resultados médios dos ensaios Panda 2 realizados em torno dos furos do SPT1 e SPT2. Estes resultados de SPTs contínuos (com avanço de $10 \mathrm{~cm}$ no furo entre ensaios) foram considerados para o cálculo das capacidades de carga, devido a semelhança com SPT3 convencional.

As Tabelas 7 e 8 apresentam os resultados das cargas admissíveis de projeto e também dos recalques dos elementos de fundação de acordo com cada método. Para melhor análise dos resultados, nas Tabelas 9 e 10 são mostradas as médias de cada um dos métodos.
Analisando os resultados da capacidade de carga obtidos pelos métodos teóricos (Terzaghi e Meyerhof) considerando os parâmetros de resistência c' e $\phi^{\prime}$ do solo nas condições natural e saturado, percebeu-se uma grande variação nos valores da capacidade de carga, demonstrando a grande influência e importância dos valores dos parâmetros adotados para o solo no cálculo, principalmente o da coesão. O uso do valor da coesão considerando o solo saturado resultou em menores valores de capacidade de carga, porém mais condizente, pois não é influenciado pela colaboração da tensão de sucção na fase de ruptura durante a realização do ensaio triaxial não saturado (condição mais próxima da realidade).

\section{TABELA 4: Resultados ensaios in situ em torno de SPT3.}

\begin{tabular}{cccc}
$\begin{array}{c}\text { Prof. } \\
(\mathbf{m})\end{array}$ & $\begin{array}{c}\text { SPT3 } \\
\text { convencional NsPT } \\
\text { (Golpes) }\end{array}$ & $\begin{array}{c}\text { qc pelo N } \text { sPT } \\
\text { via Eq. } \mathbf{1} \\
\text { (MPa) }\end{array}$ & $\begin{array}{c}\text { Panda - P3 } \\
\mathbf{q}_{\mathrm{d} 30} \\
(\mathbf{M P a})\end{array}$ \\
\hline 1,30 & 8 & 2,80 & 2,40 \\
\hline 2,30 & 10 & 3,50 & 2,15 \\
\hline 3,30 & 12 & 4,20 & 3,16 \\
\hline 4,30 & 11 & 3,85 & 3,09 \\
\hline 5,30 & 11 & 3,85 & 2,80 \\
\hline 6,30 & 11 & 3,85 & 3,91 \\
\hline 7,30 & 13 & 4,55 & 3,69 \\
\hline 8,30 & 13 & 4,55 & 2,17 \\
\hline & & & FONTE: Autoria própria.
\end{tabular}

TABELA 5: Resultados ensaios in situ em torno de SPT1.

\begin{tabular}{cccc}
$\begin{array}{c}\text { Prof. } \\
(\mathbf{m})\end{array}$ & $\begin{array}{c}\text { SPT1 contínuo } \\
\mathbf{N} \text { sPT } \\
\text { (Golpes) }\end{array}$ & $\begin{array}{c}\text { qc pelo NsPT } \\
\text { via Eq. } \\
\text { (MPa) }\end{array}$ & $\begin{array}{c}\text { Panda - P1 } \\
\text { qd30 } \\
\text { (MPa) }\end{array}$ \\
\hline 0,80 & 9 & 3,15 & 2,36 \\
\hline 1,37 & 9 & 3,15 & 2,10 \\
\hline 1,90 & 10 & 3,50 & 2,45 \\
\hline 2,46 & 8 & 2,80 & 2,20 \\
\hline 3,01 & 10 & 3,50 & 2,17 \\
\hline 3,56 & 11 & 3,85 & 2,86 \\
\hline 4,10 & 13 & 4,55 & 3,02 \\
\hline 4,66 & 11 & 3,85 & 2,73 \\
\hline 5,20 & 12 & 4,20 & 3,32 \\
\hline 5,75 & 11 & 3,85 & 3,18 \\
\hline 6,30 & 12 & 4,20 & 3,36 \\
\hline 6,90 & 10 & 3,50 & 3,32 \\
\hline 7,40 & 13 & 4,55 & 3,72 \\
\hline 7,95 & 13 & 4,55 & 3,53 \\
\hline & & & FONTE: Autoria própria.
\end{tabular}


TABELA 6: Resultados ensaios in situ em torno de SPT2.

\begin{tabular}{cccc}
$\begin{array}{c}\text { Prof. } \\
(\mathbf{m})\end{array}$ & $\begin{array}{c}\text { SPT2 contínuo } \\
\text { NsPT } \\
\text { (Golpes) }\end{array}$ & $\begin{array}{c}\text { qc pelo N } \mathbf{s P T} \\
\text { via Eq. } \mathbf{1} \\
\text { (MPa) }\end{array}$ & $\begin{array}{c}\text { Panda - P2 } \\
\mathbf{q}_{\mathbf{d} 30} \\
\text { (MPa) }\end{array}$ \\
\hline 0,86 & 8 & 2,80 & 2,76 \\
\hline 1,40 & 8 & 2,80 & 2,35 \\
\hline 1,96 & 8 & 2,80 & 2,78 \\
\hline 2,56 & 10 & 3,50 & 2,39 \\
\hline 3,10 & 10 & 3,50 & 2,84 \\
\hline 3,66 & 9 & 3,15 & 2,59 \\
\hline 4,21 & 12 & 4,20 & 3,02 \\
\hline 4,75 & 10 & 3,50 & 2,67 \\
\hline 5,30 & 10 & 3,50 & 2,95 \\
\hline 5,85 & 12 & 4,20 & 2,99 \\
\hline 6,39 & 10 & 3,50 & 2,99 \\
\hline 6,85 & 11 & 3,85 & 3,14 \\
\hline 7,40 & 12 & 4,20 & 3,76 \\
\hline 7,96 & 10 & 3,50 & 3,74 \\
\hline & & & FONTE: Autoria Própria.
\end{tabular}

\section{TABELA 7: Carga admissível e recalque da sapata pelas metodologias.}

\section{Método}

Teórico de Terzaghi $\left(c^{\prime}=107 \mathrm{kPa}\right.$ e $\left.\phi^{\prime}=30^{\circ}\right)$

Teórico de Terzaghi $\left(\mathrm{c}^{\prime}=50 \mathrm{kPa}\right.$ e $\left.\phi^{\prime}=32,5^{\circ}\right)$

Empírico - SPT1 contínuo (Eq. 6)

Empírico - SPT2 contínuo (Eq. 6)

Empírico -SPT3 convencional (Eq. 6)

Empírico - CPT 1 (Eq. 1 e 8)

Empírico - CPT 2 (Eq. 1 e 8)

Empírico - CPT 3 (Eq. 1 e 8)

Empírico - Panda 2 (P 1) (Eq. 8)

Empírico - Panda 2 (P 2) (Eq. 8)

Empírico - Panda 2 (P 3) (Eq. 8)
Carga admissível (kN)

Recalque (mm)

\begin{tabular}{cc}
7817 & 250 \\
\hline 5358 & 172 \\
\hline 813 & 26 \\
\hline 760 & 24 \\
\hline 820 & 26 \\
\hline 813 & 46 \\
\hline 760 & 43 \\
\hline 820 & 46 \\
\hline 986 & 32 \\
\hline 1064 & 34 \\
\hline 1080 & 34 \\
\hline
\end{tabular}

FONTE: Autoria própria.

\section{TABELA 8: Carga admissível e recalque da estaca pelas metodologias.}

Método

Teórico Meyerhof $\left(c^{\prime}=107 \mathrm{kPa}\right.$ e $\left.\phi^{\prime}=30^{\circ}\right)$

Teórico Meyerhof $\left(c^{\prime}=50 \mathrm{kPa}\right.$ e $\left.\phi^{\prime}=32,5^{\circ}\right)$

Semi-emp.-Décourt-Quaresma SPT 1

Semi-emp.-Décourt-Quaresma SPT 2

Semi-emp.-Décourt-Quaresma SPT 3

Semi-emp. - Aoki-Velloso SPT 1

Semi-emp. - Aoki-Velloso SPT 2

Semi-emp. - Aoki-Velloso SPT 3

Semi-emp.- Aoki-Vell. Panda 2 (P 1)

Semi-emp.- Aoki-Vell. Panda 2 (P 2)

Semi-emp.- Aoki-Vell. Panda 2 (P 3)
Carga admissível (kN)

Recalque (mm)

\begin{tabular}{ll}
\hline 4834 & 79 \\
\hline 3335 & 55 \\
\hline 613 & 10 \\
\hline 590 & 10 \\
\hline 622 & 10 \\
\hline 290 & 5 \\
\hline 315 & 5 \\
\hline 314 & 5 \\
\hline 456 & 7 \\
\hline 422 & 7 \\
\hline 512 & 8 \\
\hline
\end{tabular}

FONTE: Autoria própria. 
TABELA 9: Cargas médias admissíveis e recalque médio na sapata.

\begin{tabular}{ccc} 
Método & Carga média admissível (kN) & Recalque médio (mm) \\
\hline SPT (via NSPT pela Eq.6) & 798 & 26 \\
\hline CPT (via NSPT pela Eq. 1 e 8) & 798 & 26 \\
\hline Panda 2 (via qd3o pela Eq.8) & 1044 & 33 \\
\hline
\end{tabular}

FONTE: Autoria própria.

\begin{tabular}{ccc}
\multicolumn{2}{c}{ TABELA 10: Cargas médias admissíveis e recalque médio na estaca. } \\
\hline Método & Carga média admissível (kN) & Recalque médio (mm) \\
\hline Decourt-Quaresma (via N SPT $^{\text {) }}$ & 608 & 10 \\
\hline Aoki-Velloso (via NSPT pelas Eq. 1, 12 e 13) & 306 & 5 \\
\hline Aoki-Velloso (via qd30) & 463 & 8 \\
\hline
\end{tabular}

FONTE: Autoria própria.

$\mathrm{Na}$ análise dos resultados dos métodos teóricos em relação aos métodos empíricos e semi-empíricos, percebeu-se uma grande discrepância tanto para a fundação em sapata como para a fundação em estaca. A carga admissível pelo método teórico de Terzaghi (5.358 kN) ficou 671,4\% superior da carga média admissível da sapata pelo método empírico via SPT $(798 \mathrm{kN})$ considerado como referência neste trabalho pelo uso consagrado no país. Já para a carga admissível pelo método teórico de Meyerhof (3.335 kN) ficou $548,5 \%$ superior da carga média admissível da estaca pelo método semi-empírico de Decourt-Quaresma, via SPT $(608 \mathrm{kN})$ considerado também como referência neste trabalho pelo uso consagrado pelo meio técnico.

Segundo Cintra e Aoki (2010) no caso de fundações rasas é bastante razoável o modelo de ruptura geral de Terzaghi, entretanto a utilização de fórmulas teóricas para o cálculo da capacidade de carga de elementos de fundação por estaca apresenta grande discrepância entre as diversas proposições existentes, o que leva o descrédito do uso dos métodos teóricos em projetos sendo preteridos em prol dos métodos semi-empíricos.

Analisando os resultados da carga média admissível da sapata para os três métodos empíricos, percebeu-se ao utilizar " $k$ " de 0,20 (argila) na conversão de $\mathrm{N}_{\mathrm{SPT}}$ em $\mathrm{q}_{\mathrm{c}}$, proposto por Aoki e Velloso (1975), que o método que usou formulação para CPT coincidentemente obteve o mesmo valor $(798 \mathrm{kN})$ que o método que usou formulação direta para SPT. Já usando a formulação para CPT e entrando com o parâmetro $\mathrm{q}_{\mathrm{d} 30}$ do ensaio PANDA 2 no lugar de $q_{c}$, obteve-se valor de 1044 kN para a capacidade de carga, cerca de $31 \%$ superior à apresentada pelos outros dois métodos. Percebeuse que o valor de " $k$ ", adotado segundo o tipo de solo, influencia muito no valor da formulação do CPT para entrada com dados do SPT.

Para a estaca, a variação dos valores da carga admissível entre os métodos foi maior. $\mathrm{O}$ método de Décourt-Quaresma, que utiliza o $\mathrm{N}_{\text {SPT }}$ no cálculo da carga admissível e reconhecidamente o método mais consagrado na região para cálculo de elemento de fundação por estaca foi o que apresentou maior capacidade de carga $(608 \mathrm{kN})$, ficando superior ao valor determinado pelo método Aoki-Velloso via $\mathrm{N}_{\mathrm{SPT}}$, convertido para $\mathrm{q}_{\mathrm{c}}$, em $98,7 \%$ e ao determinado pelo método Aoki-Velloso via $\mathrm{q}_{\mathrm{d} 30}$ , utilizado no lugar de $q_{c}$ na formulação, em 31,3\%. Constatou-se que utilizando valores de $\mathrm{q}_{\mathrm{d} 30}$ do ensaio PANDA 2 aproximou-se mais do valor obtido pela metodologia de Décourt-Quaresma. Observase novamente a grande influência do valor de " $k$ " do método de Aoki e Velloso.

Dentro da discussão sobre os parâmetros k, $\alpha$, F1 e F2, outros autores como Laprovitera (1998), Benegas (1993), Monteiro (1997) contribuíram com novos valores através de retroanálise de provas de cargas realizadas em estacas. Adotando a metodologia proposta pelos referidos autores haveria alterações dos resultados deste estudo. 
Quanto à análise dos recalques percebeuse a grande variabilidade dos valores e a dependência destes com relação à carga atuante na fundação, ou seja, em casos que o carregamento foi maior, o recalque também foi maior. Entretanto, conforme demonstrado na Tabela 11, por mais que tenha variado o valor da carga admissível entre a estaca e a sapata de acordo com cada método de cálculo utilizado e variado o recalque sofrido entre a estaca e a sapata dentro do mesmo método de cálculo, a relação entre essas relações é constante e igual a cerca de $51 \%$ nos casos analisados. Isso indica que independentemente do método, para o caso em estudo a variação do recalque foi cerca de $51 \%$ da variação da carga.

\section{CONCLUSÕES}

Por meio do estudo realizado, pode-se observar que as principais divergências foram encontradas entre os valores de carga admissível calculados a partir dos métodos teóricos de Terzaghi, para a sapata, e Meyerhof, para a estaca, e os valores calculados a partir dos métodos empíricos e semi-empíricos, respectivamente.

Dentre os métodos teóricos os parâmetros de entrada de resistência do solo considerados na condição saturada resultaram em valores de capacidade de carga menos elevados comparados aos considerados na condição natural (não saturada), ficando notória a importância dos parâmetros do solo, principalmente o valor da coesão no cálculo da capacidade de carga da fundação e que pode ser muito afetado pela sucção do solo nos ensaios triaxiais CDnat.

Percebeu-se que o método de Aoki e Velloso (1975) para cálculo de capacidade de carga de estacas é muito dependente dos valores de " $\mathrm{k}$ " e " $\alpha$ " que por sua vez são muito dependentes do tipo de solo. Cabe salientar que o tipo de solo varia segundo os métodos de classificação do mesmo. Da mesma forma os parâmetros F1 e F2 também influenciam muito a capacidade e são dependentes do tipo da estaca. Como a estaca tipo hélicecontínua é mais recente no mercado, há disponível poucos estudos destes parâmetros por retroanálise de provas de carga para esse tipo de elemento de fundação.

A discrepância leva o descrédito do uso dos métodos teóricos em projetos sendo preteridos em prol dos métodos empíricos e semi-empíricos, principalmente no que tange as fundações por estacas.

Os resultados dos ensaios PANDA 2 executados foram muito semelhantes em torno de cada furo de SPT. Esta repetitividade dos resultados dos ensaios PANDA 2 dão credibilidade para o uso em dimensionamento de fundações.

TABELA 11: Relações entre cargas admissíveis ( $\left.q_{a d}\right)$ e recalques $(\rho)$ das estacas e sapatas.

\begin{tabular}{|c|c|c|c|}
\hline Ensaio e Parâmetro utilizado & $\begin{array}{c}\text { qad Estaca/qad Sapata } \\
(\%)\end{array}$ & $\begin{array}{c}\rho \text { Estaca/ } \rho \text { Sapata } \\
(\%)\end{array}$ & $\begin{array}{c}\rho / q_{\text {ad }} \\
(\%)\end{array}$ \\
\hline Via métodos teóricos (nat) & 61,83 & 31,68 & 51,2 \\
\hline Via métodos teóricos (sat) & 62,24 & 31,98 & 51,4 \\
\hline Via SPT (NsPT) & 76,21 & 39,05 & 51,2 \\
\hline Via SPT (convertendo NSPT em qc) & 38,40 & 19,68 & 51,2 \\
\hline Via Panda 2 ( $\left.q_{d} 30\right)$ & 44,41 & 24,24 & 51,2 \\
\hline
\end{tabular}


Os resultados do uso de $\mathrm{q}_{\mathrm{d} 30}$ do ensaio PANDA 2 em substituição ao valor de $q_{c}$ do ensaio CPT, nas equações dos diversos métodos empíricos e semi-empíricos de determinação da capacidade de carga admissível de elementos de fundação por sapatas e estacas mostrou-se muito promissor. Entretanto carece de mais estudos, no que tange a capacidade de carga de estacas, para os valores de $\alpha, F 1$ e F2 de Aoki e Velloso.

No estudo dos recalques, a maior variação do recalque entre a sapata e a estaca proporcionalmente à carga aplicada reforça o conhecimento de que, em geral, as estacas sofrem menos recalques do que as sapatas. Tal fato, verificado por Velloso e Lopes (2010), se deve à grande área lateral das estacas que mobilizam grande resistência por meio do atrito lateral entre solo/elemento de fundação com pouco deslocamento.

Este trabalho contribui com o meio científico a partir da aplicação dos resultados do ensaio PANDA 2, como alternativa promissora e menos convencional, em cálculos de capacidade de carga e recalques de fundações, comparando com métodos de cálculo consagrados que fazem uso dos ensaios SPT e CPT como parâmetros de entrada. Como demonstrado, os resultados tiveram pouca variação comparando diversos ensaios e métodos para o caso de aterro compactado com solo fino tropical. Entretanto, faz-se necessário mais estudos nessa linha no que tange aos diversos tipos de solos naturais.

\section{REFERÊNCIAS BIBLIOGRÁFICAS}

ABNT - Associação Brasileira de Normas Técnicas; NBR 6122 - Projeto e execução de fundações. Rio de Janeiro, 91 p., 2010.

ABNT - Associação Brasileira de Normas Técnicas; NBR 6484 - Solo - Sondagens de simples reconhecimento com SPT - Método de ensaio. Rio de Janeiro, 17 p., 2001.

ALVES, D.H. C., SILVA, A. P. da. Penetrômetro PANDA Uma nova alternativa de investigação para fundações rasas de Goiânia. Trabalho de Conclusão de Curso (Graduação em Engenharia Civil). Universidade Federal de Goiás. Goiânia, 90p. 2009.

ANGELIM, R. R. Desempenho de Ensaios Pressiométricos em Aterros Compactados de Barragens de Terra e na Estimativa de Parâmetros Geotécnicos. Tese de Doutorado, Publicação G.TD-067-1/11, Departamento de Engenharia Civil e Ambiental, Universidade de Brasília. Brasília, 271 p., 2011.

ANGELIM, R. R.; CAMPOS, C. S.; LLOBET, Y. B.; SALES, M. M. Correlação entre Ensaios SPT e Panda 2 (Penetrômetro Leve de Energia Variável) em Aterro Compactado de Barragem com Solo Tropical. In: 15 CNG - Congresso Nacional de Geotecnia e 8 CLBG - Congresso Luso-Brasileiro de Geotecnia, Porto: FEUP, ABMS e Sociedade Portuguesa de Geotecnia, 12 p., 2016(a).

ANGELIM, R. R.; CUNHA, R. P.; SALES, M. M. Determining the Elastic Deformation Modulus from a Compacted Earth Embankment Via Laboratory and Ménard Pressuremeter Tests. Soils \& Rocks (ABMS). v.39, p.285 300, 2016(b).

AZEVEDO, F. H. B.; RODRIGUES, Í. R. DE S. Estudo comparativo entre ensaios SPT e PANDA 2 em solos tropicais. Trabalho de Conclusão de Curso (graduação em Engenharia Civil), Universidade Federal de Goiás. Goiânia, 70 p., 2014.

AOKI, N.; VELLOSO, D. A. An Approximate method to estimate the bearing capacity of piles. In: Panamerican Conference on Soil Mechanics and Foundations Engineering, 5., Buenos Aires, p.367-376, 1975.

BENEGAS, H. Q. Previsões para a curva carga-recalque de estacas a partir do SPT. Dissertação (mestrado), COPPEUFRJ, Rio de Janeiro, 1993.

CINTRA, J. C.; AOKI, N. Fundações por estacas: Projeto geotécnico. Oficina de Textos; São Paulo; 96 p., 2010.

CINTRA, J. C.; AOKI, N.; ALBIERO, J. H. Fundações diretas: Projeto geotécnico. Oficina de Textos; São Paulo; 109110 p, 63-64 p., 2011.

CRUZ JR, A. J.; GITIRANA Jr., G. F. N.; SALES, M. M.; VIANA, P. M. F. Determinação do perfil de sucções a partir de amostras de SPT, e verificação de correlações entre sucção, NSPT e PANDA para solos argilosos superficiais da cidade de Goiânia. In: XVII Congresso Brasileiro de Mecânica dos Solos e Engenharia Geotécnica (COBRAMSEG2014), ABMS. Goiânia, 2014.

DÉCOURT, L; QUARESMA, A. R. Capacidade de carga de estacas a partir de valores SPT. In: Congresso Brasileiro de Mecânica dos Solos e Engenharia de Fundações, 6. Rio de Janeiro, 45-53 p., 1978.

FERREIRA, I. P.; QUIRINO, R.; SOARES, T. M. Estudo Comparativo Entre Ensaios SPT e PANDA 2 em Aterros Compactados de Barragem de Terra. Trabalho de Conclusão de Curso (Graduação em Engenharia Civil), Universidade Federal de Goiás. Goiânia, 70 p., 2013. 
LAPROVITERA, H. Reavaliação de método semi-empírico de previsão da capacidade de carga a partir de Bancos de Dados. Dissertação (Mestrado) - COPPE-UFRJ. Rio de Janeiro, 1988.

MONTEIRO, P. F. Capacidade de carga de estacas método Aoki - Velloso. Relatório interno de Estacas Franki Ltda. 1997

POULOS, H. G.; DAVIS, E. H. Elastic solutions for soil and rock mechanics. John Wiley \& Sons. New York, 1974.

SCHNAID, F. Ensaios de campo e suas aplicações à engenharia de fundações. Oficina de Textos. São Paulo, 91-105 p., 2000.

SOL SOLUTION. Panda 2 Handbook. Version 1.05, Peintamelec Ingenierie. Romagnat. France, 16-17 p., 2009.

VELLOSO E LOPES Fundações: Critérios de projeto, investigação do subsolo, fundações superficiais, fundações profundas. Oficina de Textos. São Paulo, 35 p., 37-39 p., 41 p., 44-45 p., 51 p., 56-59 p., 88 p., 241 p., 264267 p., 2010.

TEIXEIRA, A. H.; GODOY, N. S. de. Análise, projeto e execução de fundações rasas. Fundações: teoria e prática. 2ª edição. PINI. São Paulo, 227-264 p., 1998. 